\title{
On rank tests for shift detection in time series
}

\author{
Roland Fried, Ursula Gather \\ Department of Statistics, University of Dortmund, Germany
}

\begin{abstract}
Robustified rank tests, applying a robust scale estimator, are investigated for reliable and fast shift detection in time series. The tests show good power for sufficiently large shifts, low false detection rates for Gaussian noise and high robustness against outliers. Wilcoxon scores in combination with a robust and efficient scale estimator achieve good performance in many situations.
\end{abstract}

Key words: signal extraction, jumps, outliers, test resistance

\section{Introduction}

Sudden level shifts in time series, also called edges or jumps, represent important information on the course of a variable. Reliable automatic rules for level shift detection with a short time delay are needed for online analysis. A basic demand is to distinguish level shifts from minor fluctuations and short sequences of irrelevant outliers. We formalize the task using a simple additive components model, decomposing the observations $\left(Y_{t}\right)$ as

$$
Y_{t}=\mu_{t}+u_{t}+v_{t}, t \in \mathbb{Z}
$$

where $\left(\mu_{t}\right)$ is the time-varying level of the time series, which is assumed to vary smoothly with only a few sudden shifts, while $u_{t}$ is observational noise with median zero and possibly time-varying variance $\sigma_{t}^{2}$. The impulsive (spiky) noise $v_{t}$ represents an outlier generating mechanism. It is zero most of the time, but occasionally takes large absolute values.

Many filtering procedures are available for approximation of $\mu_{t}$. Linear filters such as moving averages are efficient for Gaussian noise, but they are sensitive to outliers. Running medians approximate the level $\mu_{t}$ in the center of a 
moving window $\left(y_{t-k}, \ldots, y_{t+k}\right)$ by the median, $\tilde{\mu}_{t}=\operatorname{med}\left(y_{t-k}, \ldots, y_{t+k}\right), t \in$ $\mathbb{Z}$. They offer the advantages of removing outliers and better preserving jumps (Tukey, 1977, Nieminem, Neuvo and Mitra, 1989).

Sometimes preservation of level shifts for better visualization is not enough and we want shifts to be detected automatically. There is a growing literature on robust control charts for change-point detection in time series (Davis and Adams, 2005). However, these charts typically need strong assumptions for the in-control process and the existence of a steady state, they react to several types of structural changes and they aim at a minimal average delay of detection, while sometimes the exact delay does not matter if it is too large. We however consider detection rules which are particularly designed to detect level shifts within a given time span and require only weak assumptions. Two-sample rank tests as suggested by Bovik, Huang and Munson (1986) and Lim (2006) are promising candidates for this, also because of their simplicity. The ranks of the data in a moving data window $y_{t+1}, \ldots, y_{t+k}$ of width $k$ are determined within a longer window including $h$ further observations $y_{t-h+1}, \ldots, y_{t}$ left of $t$. An upward (downward) shift between times $t$ and $t+1$ is detected if the ranks of $y_{t+1}, \ldots, y_{t+k}$ or suitable transformations of them are very large (small).

We investigate rank tests for shift detection in time series with small delays, modifying them to distinguish outlier sequences of a certain length from long-term shifts. Section 2 presents rank tests and analytic measures of their robustness. Section 3 reports a simulation study. Section 4 applies the methods to time series before some conclusions are drawn.

\section{Shift detection}

To formulate and compare rules for shift detection we assume an ideal edge of height $\delta$ after a time point $t \in \mathbb{Z}$ :

$$
\mu_{t+j}= \begin{cases}\mu, & j \leq 0 \\ \mu+\delta, & j>0\end{cases}
$$


For detection of a positive (negative) shift at time $t$ we test $H_{0}: \delta=0$ vs. $H_{1}^{+}: \delta>0\left(H_{1}^{-}: \delta<0\right)$. We restrict to a single time point in the following, considering the $n=h+k$ observations $y_{t-h+1}, \ldots, y_{t}, y_{t+1}, \ldots, y_{t+k}$ with median $\tilde{\mu}_{t}, h \geq k$. Guidelines for the choice of $h$ and $k$ are given later.

\subsection{Tests based on linear rank statistics}

Tests based on linear rank statistics have been suggested for edge detection, in particular the Wilcoxon and the median test (Bovik, Huang and Munson, 1986). Let $y_{t(1)} \leq \ldots \leq y_{t(n)}$ be the ordered observations within the window, and $r_{-h+1}, \ldots, r_{k}$ the ranks of $y_{t-h+1}, \ldots, y_{t+k}$ in this sequence. A general linear rank statistic of the most recent $k$ observations can be written as

$$
S_{+}=\sum_{j=1}^{k} a\left(r_{j}\right),
$$

with given scores $a(1), \ldots, a(n)$. The complement of $S_{+}$is denoted by $S_{-}=$ $\sum_{i=0}^{h-1} a\left(r_{-i}\right)=\sum_{i=1}^{n} a(i)-S_{+}$. The linear rank statistic

$$
L=\frac{(h+k)\left[h\left(S_{-}-\bar{a}\right)^{2}+k\left(S_{+}-\bar{a}\right)^{2}\right]}{\sum_{i=1}^{h+k}(a(i)-\bar{a})^{2}},
$$

with $\bar{a}=n^{-1} \sum_{j=1}^{n} a(j)$, is distribution-free and asymptotically $\chi_{1}^{2}$-distributed under $H_{0}$ in case of a constant variance.

The Wilcoxon test uses $a(i)=i, i=1, \ldots, n$, i.e. $S_{+}=\sum_{i=1}^{k} r_{i}$. The normalized Wilcoxon statistic $W=S_{+}-k(k+1) / 2$ takes values between zero and $k(3 k+1) / 2$ if $h=k$. The Wilcoxon scores lead to estimators and tests which are almost as effective under Gaussian noise as methods based on averages, while being more robust to deviations from this assumption (McKean, 2004).

The median test uses $a(i)=1, i=\lfloor n / 2\rfloor+1, \ldots, n$, and $a(i)=0$ otherwise. Then $S_{+}$corresponds to the number of values in $y_{t+1}, \ldots, y_{t+k}$ larger than the median of the full window and takes values between zero and $k$. The median test is regarded as reliable even in case of heavy-tailed noise. 


\subsection{Robust scale estimation}

Bovik, Huang and Munson (1986) suggest subtracting (adding) a threshold $\delta_{0}$ from $y_{t+1}, \ldots, y_{t+k}$ before applying a rank test in order to detect only large upward (downward) shifts. They recommend to choose $\delta_{0}$ larger than the noise standard deviation. If $\sigma_{t}$ is time-varying, the threshold should also vary over time, i.e. $\delta_{0}=\delta_{0}(t)$, to obtain scale-equivariant procedures. Assuming that the standard deviation is almost constant within the left part of the window, we calculate a robust estimate $\hat{\sigma}_{t}$ of $\sigma_{t}$ from $y_{t-h+1}, \ldots, y_{t}$ and chose $\delta_{0}(t)$ as a fixed multiple $d \hat{\sigma}_{t}$. We do not include $y_{t+1}, \ldots, y_{t+k}$ in the estimation of $\sigma_{t}$ to avoid masking of a shift at time $t$ because of a biased estimate of $\sigma_{t}$.

Robust scale estimators have been discussed before in the context of time series filtering (Gather and Fried, 2003). Based on these results we select some methods for further comparison. The asymptotic explosion breakdown point of the first four of them is $50 \%$, while it is only $25 \%$ for the simple and popular interquartile range. The classical MAD and IQR require calculation of sample quantiles as measures of location. The other methods are based on pairwise differences and do not need location estimates. This might be advantageous in case of a level shift since then e.g. the MAD uses a biased centering. We use the following scale estimators applied to $y_{t-h+1}, \ldots, y_{t}$ :

- The median absolute deviation about the median (Hampel, 1974):

$$
M A D_{t}=c_{h}^{M} \operatorname{med}\left(\left|y_{t-h+1}-\tilde{\mu}_{t-}\right|, \ldots,\left|y_{t}-\tilde{\mu}_{t-}\right|\right),
$$

where $c_{h}^{M}$ is a correction to achieve unbiasedness under Gaussian noise and $\tilde{\mu}_{t-}=\operatorname{med}\left(y_{t-h+1}, \ldots, y_{t}\right)$ is the median of the first $h$ observations in the window to the left of time point $t$. For large $h, c_{h}^{M} \approx c_{\infty}^{M}=1.4826$.

- The length of the shortest half (Rousseeuw and Leroy, 1988, Grübel, 1988) using the length of the shortest interval containing $50 \%$ of the data:

$$
L S H_{t}=c_{h}^{L} \min \left(y_{t(h)}-y_{t(h-m)}, \ldots, y_{t(m+1)}-y_{t(1)}\right)
$$


where $m=\lceil h / 2\rceil$ and $y_{t(1)}, \ldots, y_{t(h)}$ are the ordered values $y_{t-h+1}, \ldots, y_{t}$.

- The $Q_{h}$-estimator (Rousseeuw and Croux, 1993),

$$
Q_{h, t}=c_{h}^{Q}\left(\left|y_{t-i}-y_{t-j}\right|, 0 \leq i<j \leq h-1\right)_{\left(\left(\begin{array}{l}
\lfloor h / 2\rfloor+1 \\
2
\end{array}\right)\right)},
$$

which is approximately the first quartile of all absolute pairwise differences, and $c_{h}^{Q} \approx c_{\infty}^{Q}=2.2219$ for large $h$. The algorithm of Croux and Rousseeuw (1992) allows computation of $Q_{h, t}$ in $O(h \log h)$ time.

- The $S_{h}$-estimator (Rousseeuw and Croux, 1993) uses nested medians of the differences,

$$
S_{h, t}=c_{h}^{S} \operatorname{med}_{i} \operatorname{med}_{j \neq i}\left|y_{t-i}-y_{t-j}\right|,
$$

with $c_{h}^{S} \approx c_{\infty}^{S}=1.1926$ in large samples.

- The interquartile range (IQR) calculates the difference between the upper and the lower quartile,

$$
I Q R_{t}=c_{h}^{I}\left(y_{(0.75 h)}-y_{(0.25 h)}\right),
$$

with $c_{h}^{I} \approx c_{\infty}^{I}=0.7413$ for large $h$. We note that different definitions are available for the sample quartiles, $y_{(0.75 h)}$ and $y_{(0.25 h)}$, and that we use the interquartile range implemented in the open-source software $\mathrm{R}$.

Fig. 1 compares the finite-sample efficiencies of the methods as measured by their variances under Gaussian noise. The efficiency of the classical $M A D$ relative to the usual standard deviation is rather low and tends to $36.7 \%$. We use $M A D_{t}$ as reference in Fig. 1, so that the values exceeding $100 \%$ indicate that we can improve on the low efficiency of $M A D_{t}$ while keeping its explosion breakdown point. $Q_{h, t}$ is the most efficient robust estimator if the sample size is moderately large, say $h \geq 10$, with an asymptotic efficiency of $82.3 \%$. $S_{h, t}$ is the second most efficient estimator for most window widths $h$ with an asymptotic efficiency of $58.2 \%$. For small $h \in\{3,5,7,9\}$ it is even more efficient than $Q_{h, t}$. $L S H_{t}$ has the same asymptotic efficiency as $M A D_{t}$, but it is slightly more efficient for small Gaussian samples. $I Q R_{t}$ is not very efficient except for some small samples. 


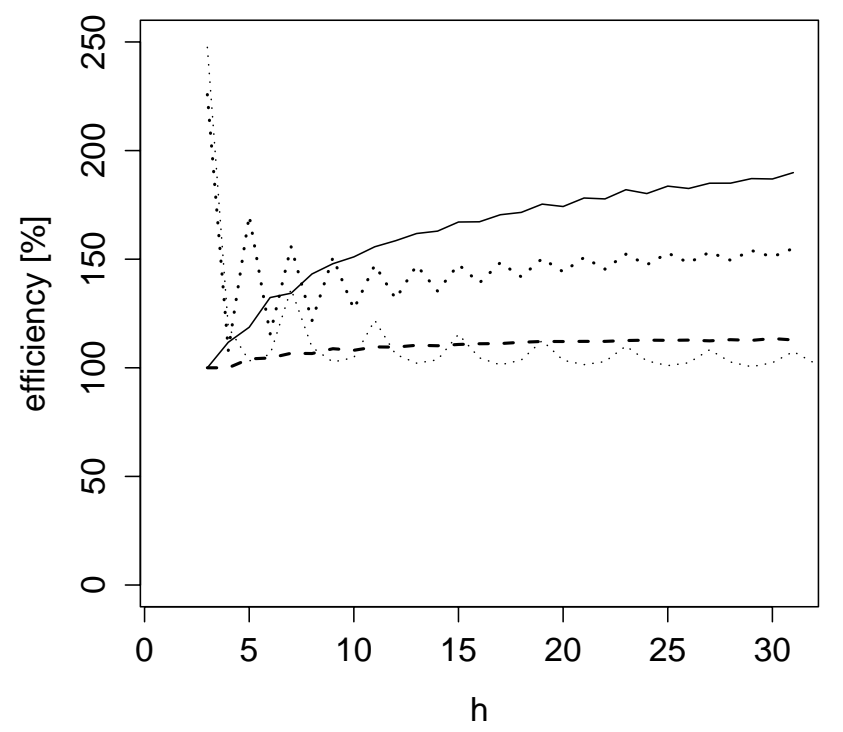

Figure 1: Efficiencies of the $L S H$ (dashed), $Q_{h}$ (solid), $S_{h}$ (bold dotted) and the $I Q R$ (dotted) relatively to the $M A D$ for different sample sizes $h$.

\subsection{Test resistances}

One reason for median filters being popular is their robustness against a substantial amount of contamination (outliers). Breakdown points are simple measures of robustness of an estimator. Given a vector of observations $\mathbf{y}=$ $\left(y_{1}, \ldots, y_{n}\right)$, the finite sample replacement breakdown point represents the minimal fraction of data set to arbitrary values that changes the estimate by any amount. This is a local concept since by definition the breakdown point depends on the sample. Fortunately, the breakdown point of the median is the same for all samples, namely $\lfloor(n+1) / 2\rfloor / n$, converging to $50 \%$ with increasing $n$.

Of course, the outcome of the test should not be determined by a few outliers. Let $U_{m}(\mathbf{y})$ denote a neighborhood of $\mathbf{y}$ consisting of all data vectors $\mathbf{z}=\left(z_{1}, \ldots, z_{n}\right)$ with $z_{i} \neq y_{i}$ for at most $0 \leq m \leq n$ positions. Let $\phi$ 
be the decision function of the test, $\phi(\mathbf{z})=1$ and $\phi(\mathbf{z})=0$ representing rejection and non-rejection of the null hypothesis, respectively. Ylvisaker (1977) suggests the global concept of resistance of a test to acceptance and rejection. Deviating slightly from his definition, these quantities are

$$
\begin{aligned}
\epsilon_{A} & =\frac{1}{n} \min \left\{m \geq 0: \sup _{\mathbf{y} \in \mathbb{R}^{n}} \inf _{\mathbf{z} \in U_{m}(\mathbf{y})} \phi(\mathbf{z})=0\right\}, \text { and } \\
\epsilon_{R} & =\frac{1}{n} \min \left\{m \geq 0: \inf _{\mathbf{y} \in \mathbb{R}^{n}} \sup _{\mathbf{z} \in U_{m}(\mathbf{y})} \phi(\mathbf{z})=1\right\} \text {, respectively. }
\end{aligned}
$$

Ylvisaker considers modifications to occur only at the last positions for simplification. For unstructured data the resulting resistances are usually identical to those obtained when modifications are allowed at arbitrary positions as it is done here. The resistances depend on the significance level but not on the sample since the supremum (infimum) over all possible data $\mathbf{y}$ is taken, i.e. the test breaks down if it does so for any sample. We cannot use a worst case data set in the definition since for $\mathbf{y}$ with $\phi(\mathbf{y})=0$ we always have $\inf _{\mathbf{z} \in U_{m}(\mathbf{y})} \phi(\mathbf{z})=0$. Another, computationally very expensive approach to overcome the dependence on the sample is expected resistance (Coakley and Hettmansperger, 1992).

We apply the concept of resistances to search for tests which can detect a level shift in spite of nearby outliers. For this we analyze two-sample tests comparing the levels in the left- and the right-hand window. Denote the corresponding samples by $y_{11}, \ldots, y_{1 h}$ and $y_{21}, \ldots, y_{2 k}$, respectively. It is easy to see that the test based on the difference of the arithmetic means, $\bar{y}_{2}-\bar{y}_{1}$, applied in case of a known scale $\sigma$ posses resistance to rejection and to acceptance both equal to $1 / n$ at any significance level $\alpha$ with $n=h+k$. This test can easily be mislead since a single outlier can either cause incorrect detection of a shift or mask a shift so that it is not detected.

The two-sample t-test for the situation of an unknown scale also has got resistance to acceptance equal to $1 / n$. Moving one observation is always sufficient to get a difference of the arithmetic means of zero: a single outlier can mask a level shift of any size for the t-test. The resistance to rejection is 
more difficult to derive. To increase the squared test statistic by an arbitrary amount, so that the p-value becomes zero and thus smaller than any $\alpha$, needs modification of at least $\min \{h, k\}$ out of $n$ observations. Nevertheless, fewer modifications can have a large, albeit bounded effect, and can make the test statistic exceed the critical value at certain significance levels $\alpha$.

Hence, the number of outliers a test for shift detection can deal with without becoming unreliable does not only depend on the window widths $h$ and $k$, but also on $\alpha$. In the following we tune all tests to obtain $\alpha=0.1 \%$ in case of Gaussian noise, so that we expect to detect a level shift incorrectly only once in 1000 observations. We note that for ordinary rank tests this excludes very small choices of $h$ and $k$ since they cannot obtain such a small false detection rate in small samples. Upper limits for $h$ and $k$ are imposed by the time periods in which the level $\mu_{t}$ can be assumed to be constant, and especially for $k$ by the admissible time delay of detection in online analysis. We choose windows of the same width $h=k$ for simplicity. This gives also some protection against unequal variances in the two windows (Staudte and Sheather, 1990).

Table 1 shows critical values for the Wilcoxon statistic $W$ and for the number $S_{+}$of observations larger than the median all corresponding to an approximate value of $\alpha \approx 0.1 \%$ in two-sided testing. The exact test sizes as well as the corresponding resistances to rejection and to acceptance are given as well. The resistances to rejection are hard to derive and give lower bounds instead, calculated for a situation with the observations from the two windows in alternating order. These bounds are satisfactory for our purpose since the resistances to acceptance are much lower and thus more critical.

We need to choose $h=k=7$ to obtain $\alpha=0.1 \%$ if $h=k$. For the median test $h=k=10$ is necessary to obtain a resistance to acceptance of $2 / n$ and to prevent that a shift of any size can be masked by a single outlier. The resistances of the Wilcoxon test are smaller, we even need $h=k=11$ for this. The small resistances look surprising but are simply due to the fact that for $h=k \leq 9$ all observations in the right-hand window need to be 
Table 1: Critical values $C$, test sizes $\alpha$ (in \%), lower bounds for the resistances to rejection (RR) and exact values of the resistances to acceptance (RA) for the median (top) and the Wilcoxon test (bottom) and different values of $h=k(n=h+k)$.

\begin{tabular}{lcccccccccc}
$k$ & 6 & 7 & 8 & 9 & 10 & 11 & 12 & 13 & 14 & 15 \\
\hline$C$ & 6 & 7 & 8 & 9 & 9 & 10 & 11 & 11 & 12 & 13 \\
$\alpha$ & .216 & .058 & .016 & .004 & .109 & .035 & .011 & .120 & .042 & .015 \\
$n \cdot \mathrm{RR}$ & 5 & 6 & 7 & 8 & 8 & 9 & 10 & 10 & 11 & 12 \\
$n \cdot \mathrm{RA}$ & 1 & 1 & 1 & 1 & 2 & 2 & 2 & 3 & 3 & 3 \\
\hline$C$ & 36 & 48 & 61 & 76 & 91 & 108 & 127 & 148 & 169 & 191 \\
$\alpha$ & .216 & .117 & .093 & .078 & .105 & .106 & .089 & .10 & .10 & .10 \\
$n \cdot \mathrm{RR}$ & 5 & 5 & 5 & 6 & 6 & 6 & 6 & 7 & 7 & 7 \\
$n \cdot \mathrm{RA}$ & 1 & 1 & 1 & 1 & 1 & 2 & 2 & 2 & 2 & 3
\end{tabular}

either larger or smaller than those in the left one for shift detection. For the median test we have the formula $n \cdot R A=k-C+1$.

\subsection{Robustified rank tests}

Given the structural weakness of both the Wilcoxon and the median test observed above we robustify rank tests aiming at higher resistances. We fix the critical values for $W$ and $S_{+}$to be maximal under the restriction that we always want to detect an upward (downward) shift if, after subtracting (adding) a threshold, the largest (smallest) $\lfloor(k+1) / 2\rfloor$ observations are in the right-hand window. Putting things the other way round, this gives us a chance to detect an upward (downward) shift even if almost half of the observations $(\lfloor(k-1) / 2\rfloor$ out of $k)$ in the right-hand window are extremely small (large). We thus fix critical values for $W$ and $S_{+}$guaranteeing high resistances without taking the desired false detection rate $\alpha$ into account. For $h=k=7$ e.g., we choose 28 and 4 , respectively. Then $\alpha$ is regulated by subtracting (adding) a suitable multiple $\delta_{0}(t)=d \hat{\sigma}_{t}$ of one of the robust 
scale estimates presented in Section 2.2 from the observations in the righthand window when testing for an upward (downward) shift. We determine suitable constants $d$ achieving $\alpha=0.1 \%$ in simulations. Two one-sided tests are performed at each time point to detect upward and downward shifts.

The resistance to acceptance of these tests is at least $\min \left\{\lfloor(k+1) / 2\rfloor / n, \epsilon^{*} h / n\right\}$, with $\epsilon^{*}$ being the explosion breakdown point of the scale estimator $\hat{\sigma}$ : Let $\mathbf{y}_{1}=\left(y_{11}, \ldots, y_{1 h}\right)^{\prime}$ be arbitrary observations in the left-hand window. When moving less than $h \cdot \epsilon^{\star}$ of them, the resulting scale estimate is still bounded, say smaller than $M\left(\mathbf{y}_{1}\right)$. Let now the observations in the right-hand window $\mathbf{y}_{2}=\left(y_{21}, \ldots, y_{2 k}\right)^{\prime}$ be such that $\min \left(y_{21}, \ldots, y_{2 k}\right)>\max \left(y_{11}, \ldots, y_{1 h}\right)+$ $d M\left(\mathbf{y}_{1}\right)$. By construction, the modified values, which we obtain from $\mathbf{y}_{2}$ after subtracting $d$ times the scale estimate for the left-hand window, are all larger than all values in $\mathbf{y}_{1}$, even when modifying at most $h \cdot \epsilon^{\star}-1$ observations in the left-hand window before. If we then move at most $\lfloor(k-1) / 2\rfloor$ observations in $\mathbf{y}_{2}$, by construction there are still enough unmodified observations in $\mathbf{y}_{2}$ to detect a shift.

The resistance to rejection of the robustified tests is at least $\lfloor(k+1) / 2\rfloor / n$. Let $\mathbf{y}_{1}=\left(y_{11}, \ldots, y_{1 h}\right)^{\prime}$ be arbitrary, with all values different, and all values of $\mathbf{y}_{2}=\left(y_{21}, \ldots, y_{2 k}\right)^{\prime}$ in the median interval of $\mathbf{y}_{1}$ for $k$ even, and in between the neighbors of the median of $\mathbf{y}_{1}$ for $k$ odd. The tests will not reject the null for any sample obtained from this one by less than $\lfloor(k+1) / 2\rfloor$ modifications.

\section{$3 \quad$ Monte Carlo experiments}

We perform a simulation study to compare small-sample properties of the different detection rules introduced in Section 2. We use the components model (1) and analyze the behavior at a single time point $t$. The suitable choice of the window widths $h$ and $k$ depends on the application, i.e. on the situations a filtering procedure needs to handle. For resisting patches of subsequent outliers we must choose $h$ and $k$ sufficiently large, while upper limits are imposed by the duration of periods in which the level can be 
assumed to be approximately constant, and for $k$ by the admissible time delay. For simplicity, we concentrate on windows with the same width $h=k$, and use the same $k$ for all detection rules to achieve the same delay. The level is assumed constant within both windows, i.e. we consider ideal edges, and the observational noise $\left(u_{t+j}\right)$ is standard Gaussian if not stated otherwise. The basic experiments are performed for $h=k=7$, assuming the level to be constant only for short time horizons. We then repeat the experiments for $h=k=6$, for which the ordinary rank tests had to be designed as liberal, and for $h=k=15$ to verify the results.

\subsection{Power for different types of noise}

First we compare the power of the tests for detecting shifts of different heights $\delta=0.5,1, \ldots, 10$ in standard Gaussian white noise. We generate 10000 windows for each height and derive the power as the percentage of cases in which a shift is detected, see Figure 2 for $h=k=7$. The ordinary Wilcoxon test shows almost the same power as the t-test. The robustified rank tests are less powerful, with the median tests being worse than the Wilcoxon tests. $S_{h, t}$ leads to the largest power if $h=k=7$, followed by $I Q R_{t}$ and $Q_{h, t}$. The tests based on $M A D_{t}$ or $L S H_{t}$ are the least powerful. This ordering corresponds to the factor $d$ in the threshold $d \hat{\sigma}_{t}$ as it is the smallest for $S_{h, t}$ and the largest for $M A D_{t}$. This in turn can be explained by the efficiencies of the estimators which is highest for the $S_{h}$ and smallest for the $M A D$ if $k=7$, see Fig. 1 . For $h=k=6$ and $h=k=15, Q_{h, t}$ leads to the largest power in agreement with its high efficiency. $S_{h, t}$ and $I Q R_{t}$ follow, while $L S H_{t}$ and $M A D_{t}$ again lead to the least powerful tests.

Identical measurements due to e.g. rounding yield a problem for robust scale estimators. A simple solution is 'wobbling', i.e. adding random noise to the observations. We generate data as before and round all observations to the nearest .5. The observational noise thus takes on one of the nine values $-2,-1.5, \ldots, 1.5,2$ with more than $95 \%$ probability. We then add uniform

$U(-0.25,0.25)$ noise to all values to recover the full range. The results do 
not appear sensitive to such changes in the data, i.e., wobbling allows to maintain the properties of the methods almost completely.

There are also only small changes in the ordering of the methods when generating the noise from a $t$-distribution with three degrees of freedom. The differences between the t-test and the rank tests are somewhat reduced as compared to the Gaussian situation if $h=k=6$ or $h=k=7$, while for $h=k=15$ robustified Wilcoxon tests perform almost as well as the t-test, and the ordinary Wilcoxon test does even better, see Figure 2.

\subsection{Single outlier}

Next we check the sensitivity of the methods against a single outlier, starting with the false detection rate. We replace one of the observations by an additive outlier of size $s \in\{1,2, \ldots, 20\}$ and calculate the error of first kind from 20000 simulation runs for each $s$, see Fig. 3 for $h=k=7$. The error rate of the t-test decreases to zero since the limit of the squared test statistic is 1 as the outlier size tends to infinity. An outlier increases the false detection rate of the rank tests to up to $0.2 \%-0.3 \%$ while it is in the righthand window, with $Q_{h, t}$ and $L S H_{t}$ providing slightly more stable results than the other methods. When the outlier enters the left-hand window it still increases the false detection rates of the robustified rank tests, but to a smaller amount than before. This continuing increase might be due to a small effect on the robust scale estimates in small samples. The influence of a single outlier decreases with the window width as could be expected.

We also investigate the effect of an outlier on the power of the procedures in case of a shift of height $10 \sigma$ and $h=k=7$. We replace either one observation in the left-hand window by a positive outlier of size $20 \sigma$, or one in the right-hand window by a negative one, see Fig. 3 for the powers from 10000 simulations runs. The power of the two-sample t-test approaches zero as the outlier size becomes larger than the height of the shift. For the 

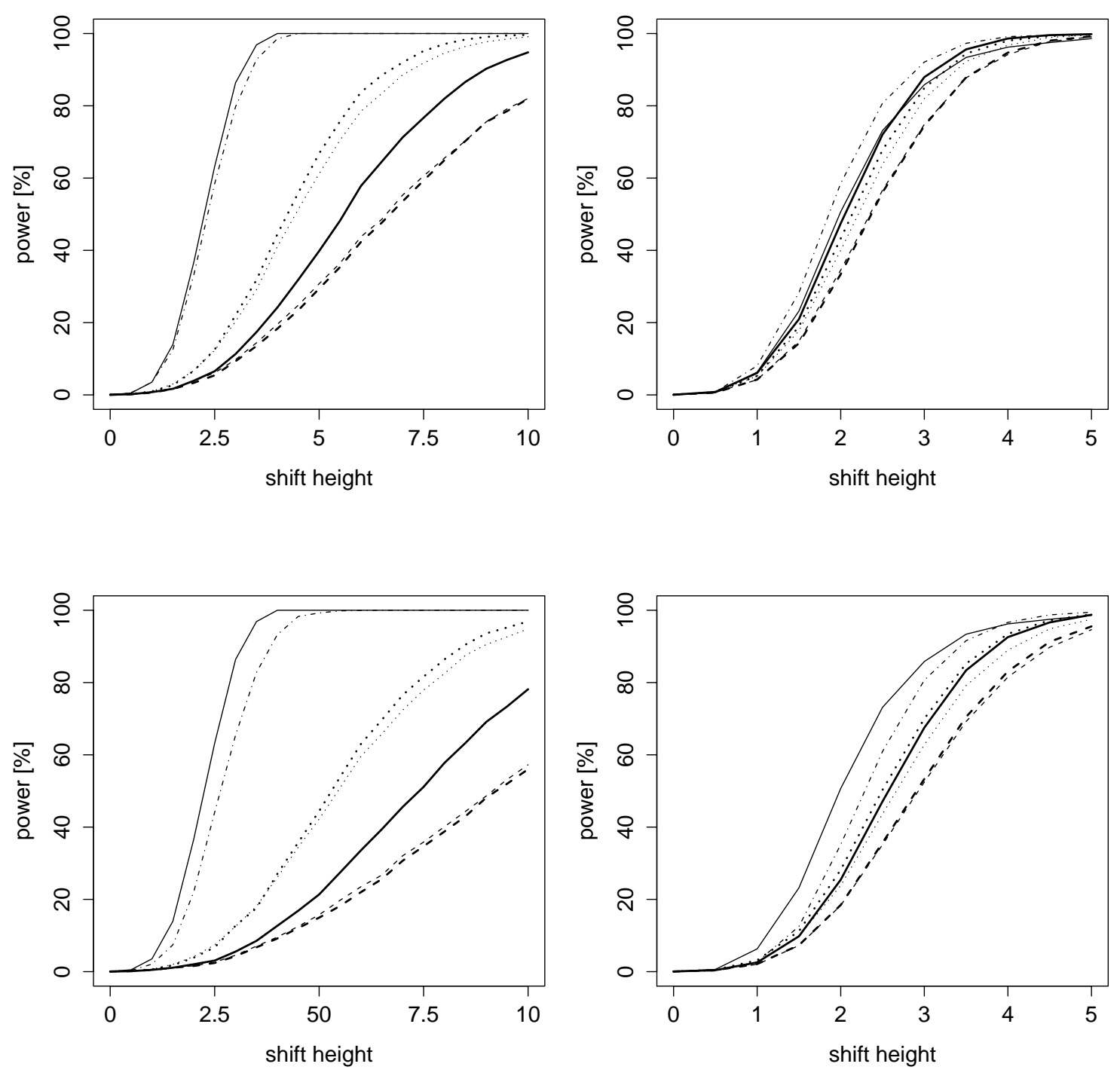

Figure 2: Power for shifts of different heights in case of Gaussian noise with $h=k=7$ (left) and $t_{3}$-noise with $h=k=15$ (right), Wilcoxon (top) and median tests (bottom): $\delta_{0}=0$ (dash-dot), MAD (dashed), IQR (dotted), $L S H$ (bold dashed), $Q_{h}$ (bold solid) and $S_{h}$ (bold dotted); the t-test (solid) is included for comparison. 

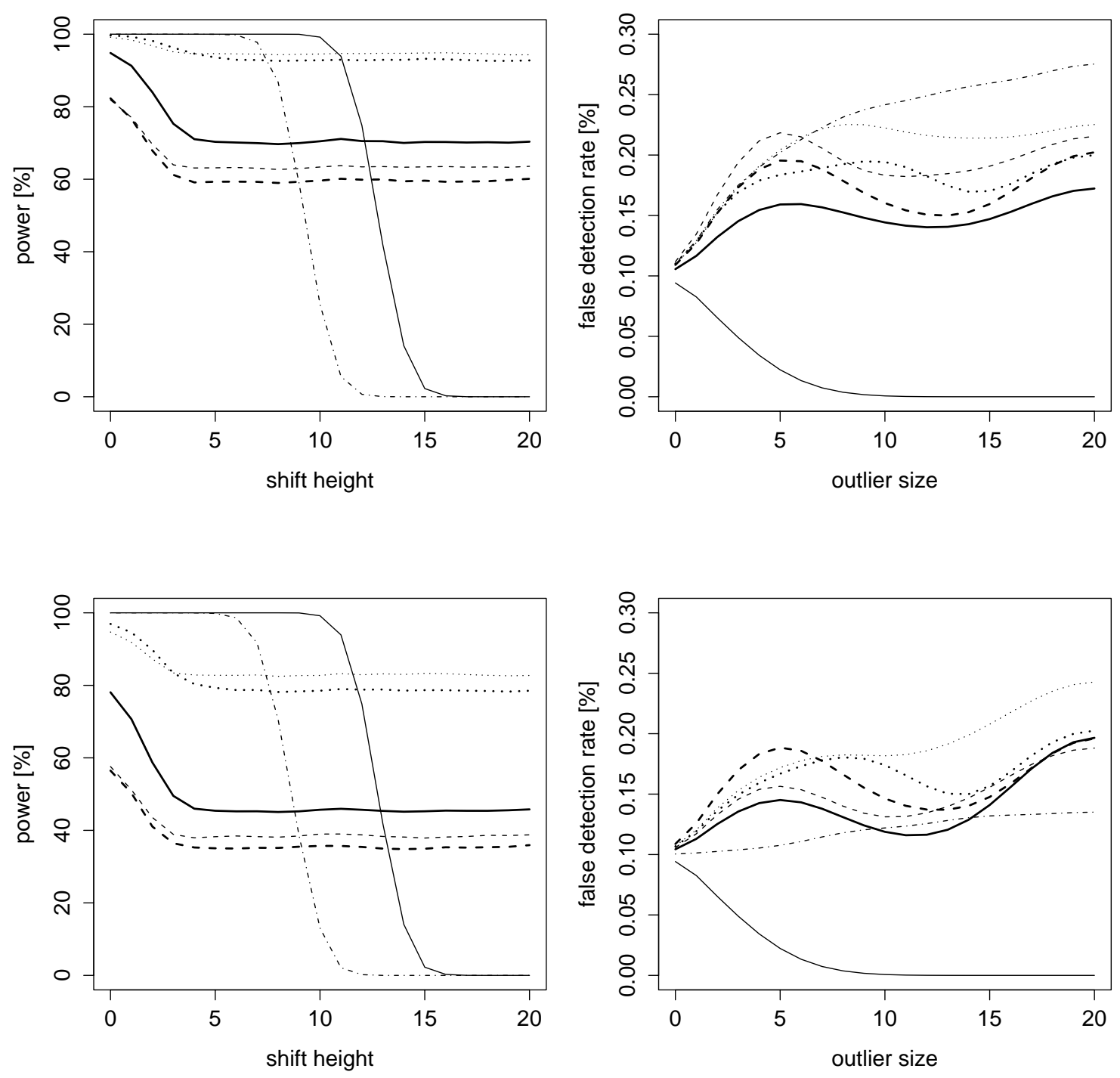

Figure 3: Power for a shift of size $10 \sigma$ in case of an outlier in the left window (left) and false detection rate in case of an outlier in the right window (right), Wilcoxon (top) and median tests (bottom), $h=k=7: \delta_{0}=0$ (dash-dot), $M A D$ (dashed), IQR (dotted), LSH (bold dashed), $Q_{h}$ (bold solid) and $S_{h}$ (bold dotted); t-test (solid). 
ordinary rank tests this happens even earlier. A few outliers can prevent shift detection when using ordinary rank tests with a short window. These findings confirm the relevance of the resistances to acceptance given in Subsection 2.3 in practice.

The power of the robustified rank tests remains almost unaffected by an outlier in the right window, even in case of small samples. An outlier in the left window, i.e. just before the shift, causes a moderate loss of power. This effect might again be due to a slight increase of the scale estimate. It stops increasing as the outlier size becomes larger than $4 \sigma$.

The results for $h=k=6$ and $h=k=15$ are similar to those above. Choosing longer windows reduces the loss of power of the two-sample t-test and the ordinary rank tests, but such choices are not always possible and also lead to results inferior to those of robustified rank tests with robust scales.

\subsection{Multiple outliers}

Next we investigate the performance in case of multiple outliers. Starting from a steady state, we insert an increasing number of outliers of the same size into one window. Fig. 4 shows the detection rates for $h=k=7$ obtained from 10000 simulations runs each. The t-test needs at least six (out of seven) deviating observations to detect a shift. This resistance is not desirable since a situation with five shifted observations is closer to a shift with two outliers in a sense than to a steady state with five outliers. Two outliers of the same size as the shift can mask a shift for the t-test, like one outlier larger than the shift. For the ordinary rank tests all observations must be shifted to guarantee detection, see Subsection 3.2. The robustified rank tests indicate a shift of size $10 \sigma$ reliably if the outliers are in the right window. The methods are almost unaffected if less than half of the observations are

deviating (three out of seven), and mostly indicate a shift whenever more than half of the observations are shifted.

The situation gets more difficult when the outliers are in the left-hand window, before the shift. All tests rarely detect a shift in case of four outliers 
then. Only $S_{h, t}$ allows detection with high power in case of five deviant observations, a situation which could arise from two outliers occurring just before a shift. The other scale estimators yield a smooth increase of the detection rate with increasing number of deviations. Generally, the most efficient scale estimators result in the largest power if the majority of the observations is shifted. Only the tests using $I Q R_{t}$ perform as poorly as the t-test, since $I Q R_{t}$ increases strongly if two to five out of seven observations are deviating. The robustified rank tests show higher detection rates in case of four or five observations shifted by a larger amount $20 \sigma$ in the lefthand window, but only $Q_{h, t}$ leads to a good power in case of four shifted observations, thus allowing the construction of robustified rank tests with consistent behavior for huge shifts.

We find similar results for $h=k=6$. The robustified rank tests detect a shift if more than three observations in the right-hand window are shifted, with $Q_{h, t}$ yielding the largest powers according to its high efficiency. A shift is rarely detected by any method in case of up to three outliers in the left- or the right-hand window. At most one outlier before a shift is tolerated with high power since all scale estimators are affected by two outliers within six observations.

For $h=k=15$ we consider a smaller shift of size $6 \sigma$, see Fig. 5. The detection rate of the t-test increases from six to nine shifted observations (out of fifteen). The robustified rank tests are again rather consistent if the outliers are in the right window. They resist about five outliers well and indicate a shift reliably in case of eight outliers, where again the most efficient estimators yield the most powerful tests. If the outliers are in the left window only $Q_{h, t}$ leads to a reasonable power in case of eight shifted observations. In general, tests using the $I Q R_{t}$ can be severely mislead by outliers just before a shift because of its rather low breakdown point. 

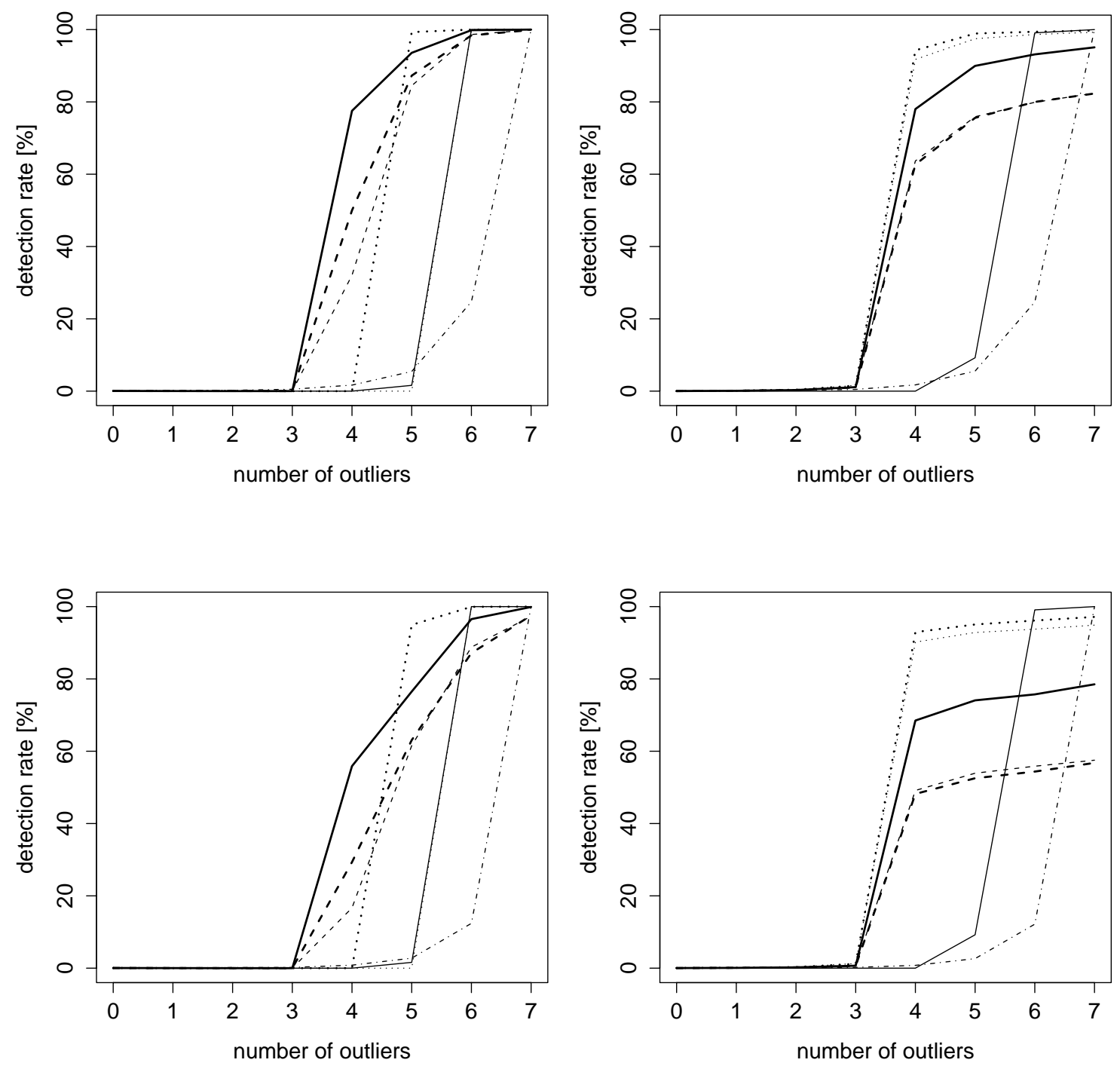

Figure 4: Percentage of detected shifts in case of an increasing number of observations shifted by $20 \sigma$ in the left or by $10 \sigma$ in the right window, Wilcoxon (top) or median tests (bottom), $h=k=7: \delta_{0}=0$ (dash-dot), $M A D$ (dashed), IQR (dotted), LSH (bold dashed), $Q_{h}$ (bold solid), $S_{h}$ (bold dotted); t-test (solid). 

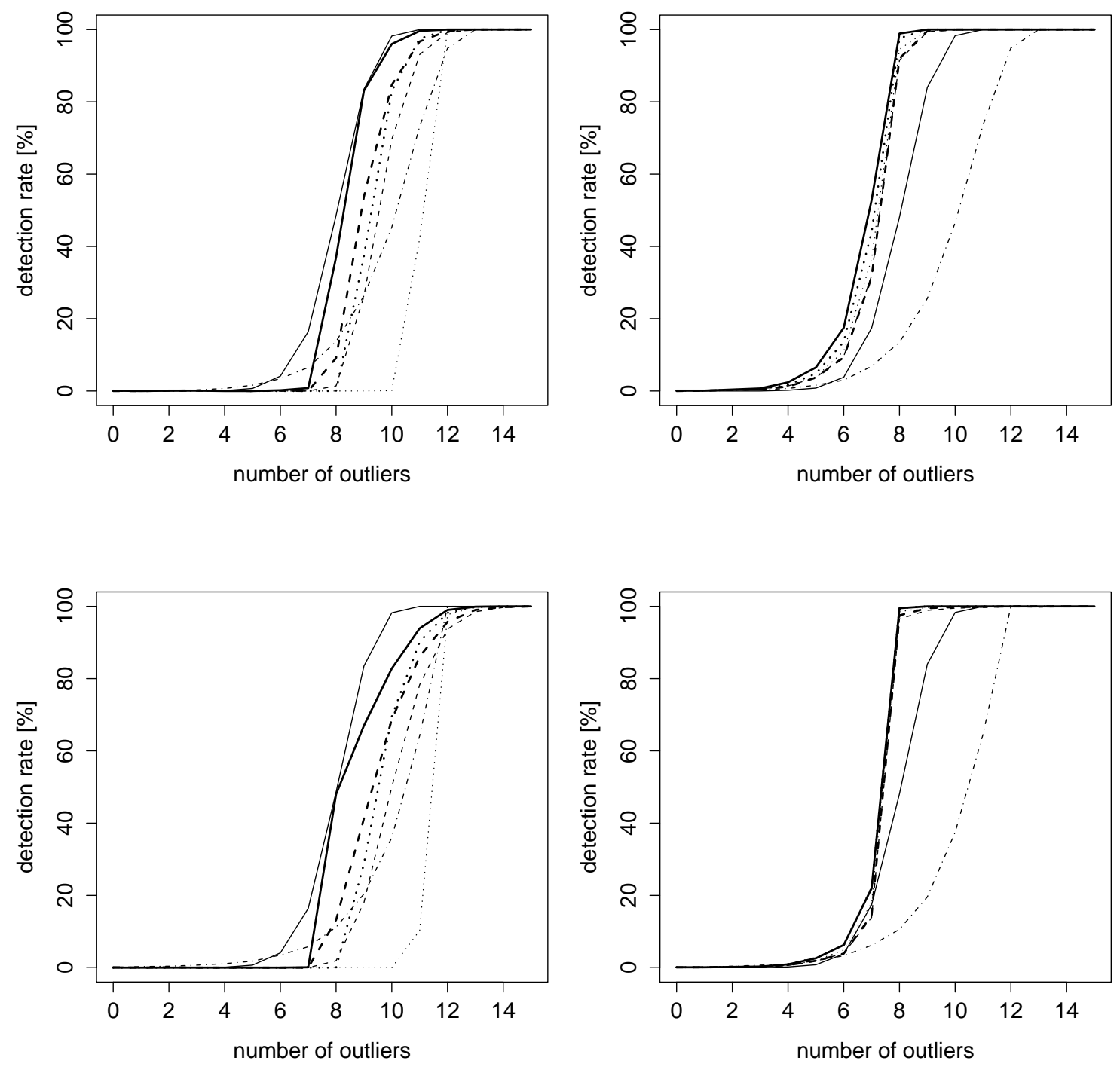

Figure 5: Percentage of detected shifts in case of an increasing number of observations shifted by $6 \sigma$ in the left or the right window, Wilcoxon (top) and median tests (bottom), $h=k=15: \delta_{0}=0$ (dash-dot), MAD (dashed), $I Q R$ (dotted), $L S H$ (bold dashed), $Q_{h}$ (bold solid), $S_{h}$ (bold dotted); t-test (solid). 


\subsection{Autocorrelated data}

In many applications we are confronted with autocorrelations. To investigate the rules in such situations we generate the observational noise from AR(1)models, $u_{t}=\phi u_{t-1}+\epsilon_{t}$, with the innovations $\epsilon_{t}$ forming standard Gaussian white noise $N\left(0, \tau^{2}=1\right)$, and lag-one correlation $\phi \in\{-0.9,-0.8, \ldots, 0.9\}$. $\mathrm{AR}(1)$-models are a convenient choice for autocorrelations.

The error rates of all methods increase with increasingly positive autocorrelations, see Fig. 6. The more powerful methods suffer from larger increases. In particular, the t-test and the ordinary rank tests show rejection rates up to $17 \%$ and $7.4 \%$ (Wilcoxon) and $5.3 \%$ (median test), respectively, in case of $\phi=0.9$ and $h=k=7$. The robustified rank tests show more moderate increases. Increasing the window width does not remove these effects.

For an investigation of the power of the methods we fix the shift to ten times the standard deviation $\sigma=\tau / \sqrt{1-\phi^{2}}$ of the observations, see Fig. 6. There is a small loss of power for negative and an increase of power for positive $\phi$, but as we have seen before the false detection rates of the methods also increase in the latter case. $Q_{h, t}$ outperforms $S_{h, t}$ and $I Q R_{t}$ in case of a large negative $\phi$. The ordering among the methods remains constant otherwise, also when varying the height of the shift for a fixed $\phi=0.6$.

\section{Application}

For further illustration we apply the procedures to a time series of length $N=500$ generated from the components model (1), see Figure 7. The underlying signal $\left(\mu_{t}\right)$ is the blocks function (Donoho and Johnstone, 1994), a benchmark example for edge-preserving smoothing. This signal is overlaid by Gaussian noise with time-varying, signal dependent standard deviation

$\sigma_{t}=1+\left|\mu_{t}\right| / 20$. We replace a total of 24 observations by twelve isolated and six pairs of outliers of size 8 . We do not only want to preserve the edges, but 


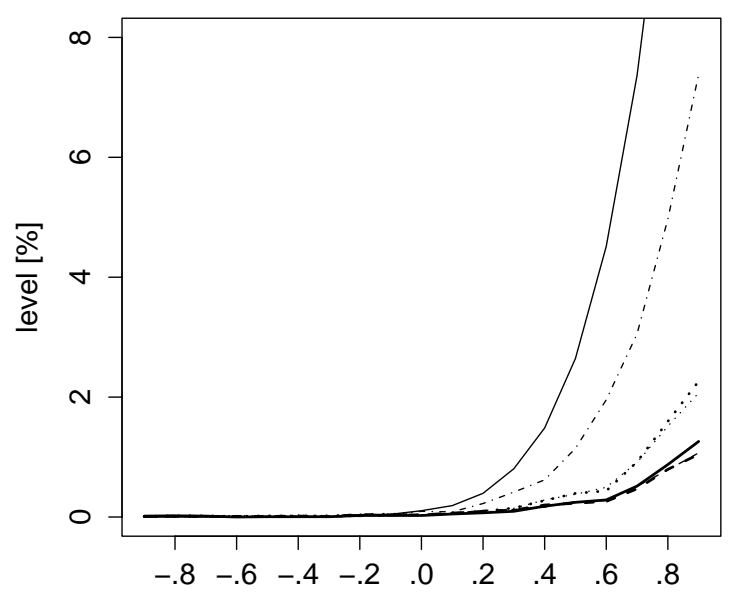

lag-one autocorrelation

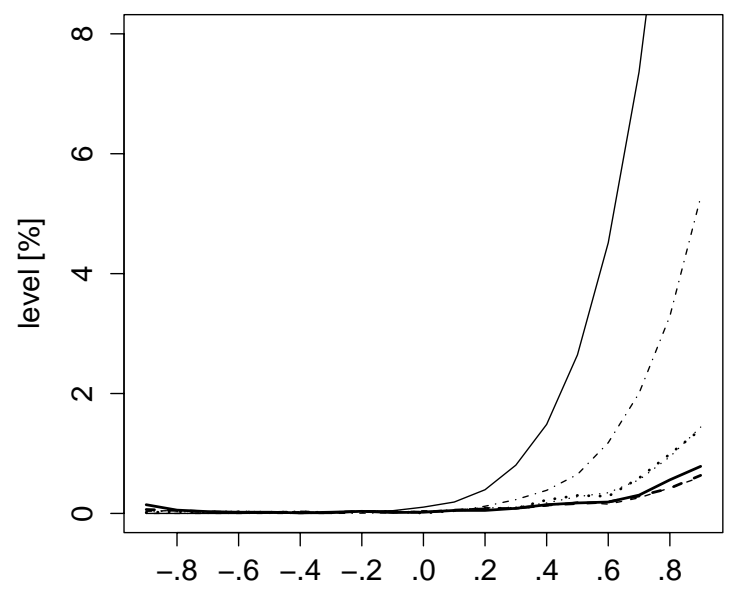

lag-one autocorrelation

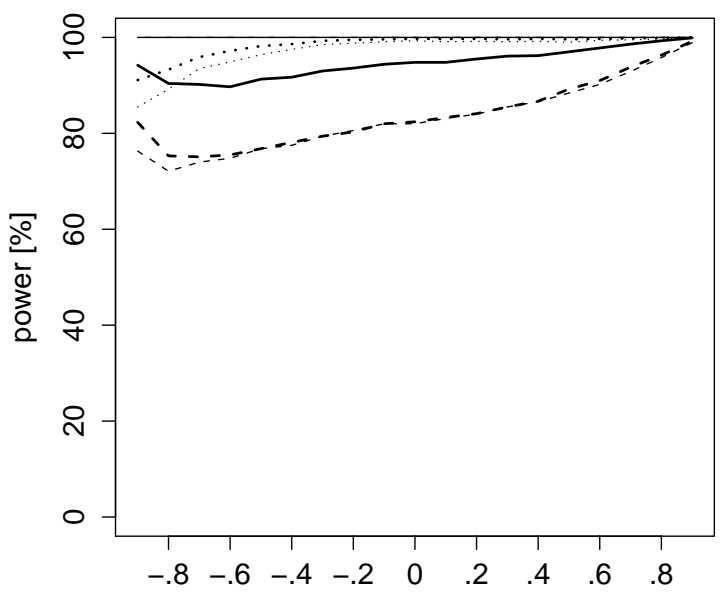

lag-one autocorrelation

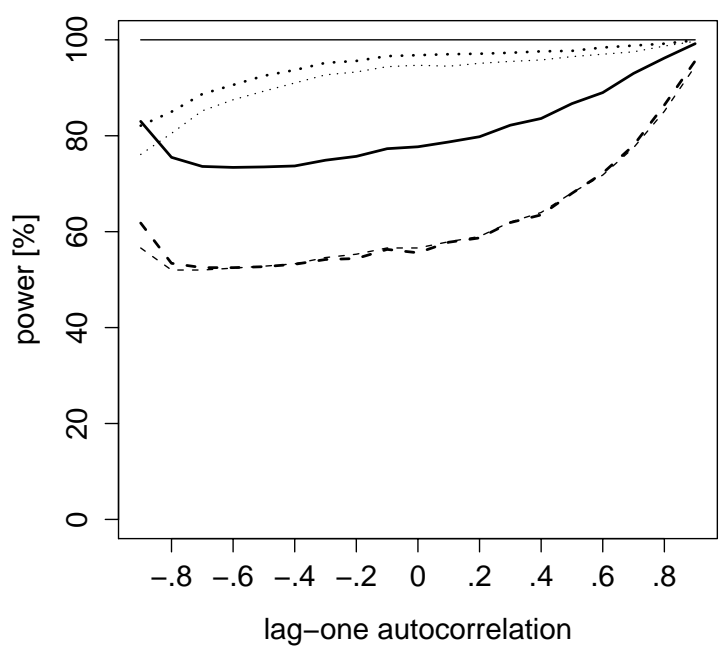

Figure 6: False detection rate (left) and power for a $10 \sigma$-shift (right) in dependence on $\phi$, Wilcoxon (top) and median tests (bottom), $h=k=7$ : $\delta=0$ (dash-dot), $M A D$ (dashed), IQR (dotted), $L S H$ (bold dashed), $Q_{h}$ (bold solid), $S_{h}$ (bold dotted); t-test (solid). 
detect them with a small time delay and without unnecessary false alarms.

We filter this time series by a running median with window width 15 . The rules for shift detection investigated above are applied to improve the results, analyzing at each time point $t$ the ranks of $y_{t+1}, \ldots, y_{t+7}$ as compared to $y_{t-7}, \ldots, y_{t-1}$, i.e. we choose the window widths $h=k=7$. Detection of a shift allows to take some appropriate action. We calculate a simple estimate of the time point at which the level has shifted as follows: if at time $t$ a shift is detected without a previous alarm at $t-1$, a candidate time point for the shift is right before the first $t+j, j>0$, for which $y_{t+j}$ is closer to the median $\tilde{\mu}_{t+}$ of $y_{t+1}, \ldots, y_{t+7}$ than to the median $\tilde{\mu}_{t-}$ of $y_{t-7}, \ldots, y_{t-1}$. Instead of the median of the full window we then take the median of the left-hand window up to the candidate time $t+j$, verifying (and possibly changing) the candidate time point in each step. From time point $t+j$ on we then use the median of the current right-hand window until returning to the standard procedure at time $t+j+4$.

As we have seen before false alarms are rarely triggered by any of the rules. Accordingly, the results of all methods are identical during a steady state without shift. Fig. 7 depicts several parts of the series in which one or several shifts occurred along with some filter outputs. The shifts at times 50 and 106 are neither detected by the t-test nor by the ordinary rank tests. In the first case the reason is masking by the pair of outliers right after the shift. The filters applying one of these rules therefore do not adapt early enough to the shift or smear it slightly like the running median without detection rules. The latter additionally smooths the shifts at times 66 and 75 somewhat. The robustified median tests, just like the Wilcoxon tests except for $S_{h, t}$ and $L S H_{t}$, do not detect the shift at $t=75$ before this time point. Similarly, the ordinary rank tests detect the shifts at $t=325$ and $t=380$ rather late.

In general, the robustified Wilcoxon test using $S_{h, t}$ gives the best results as could be expected in view of Section 3. We note that a smoother filter output could be obtained easily using one of these procedures in combination with exponential smoothing between the identified level shifts. 

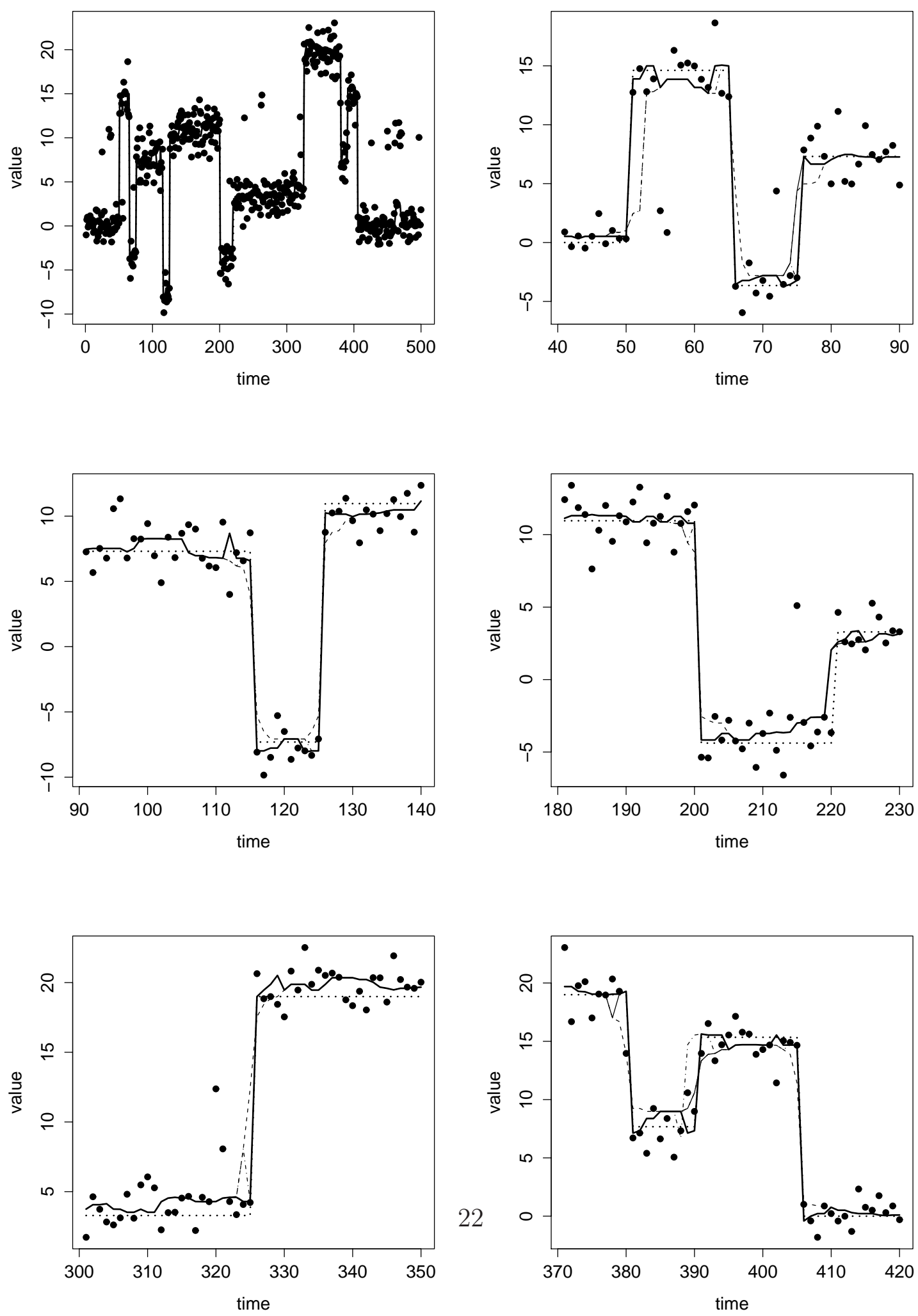

Figure 7: Time series (bold dots) generated from the blocks function (bold dotted) overlaid by time-varying noise and some time periods: running median (dashed), ordinary rank tests (dash-dot), robustified Wilcoxon test with $Q_{h}$ (solid) and with $S_{h}$ (bold solid). 


\section{Conclusions}

Tests based on linear rank statistics, in particular the Wilcoxon and the median test, have been suggested repeatedly for robust edge detection in images or time series. However, although they are insensitive to deviations from Normality, they nevertheless can be mislead by a few outliers masking a shift.

Modification by a threshold has been suggested with the aim of detecting only relevantly large shifts. This idea can be used for robustification of rank tests, using a multiple of a robust scale estimate for the threshold. The resulting robustified rank tests are no longer distribution-free, but they resist outliers much better and distinguish reliably large level shifts from a steady state even if almost one quarter of the observations included in the testing are outlying. A threshold additionally allows to reduce the false detections caused by positive autocorrelations without eliminating them completely.

A robustified median test in combination with $Q_{h, t}$ was already used by Fried (2004). Based on our results we can indeed recommend the $Q_{h^{-}}$, or the $S_{h}$-estimator for certain window widths since highly efficient scale estimators yield the highest probabilities of shift detection within a short time delay specified before. We have shown that the power can be further increased without loosing robustness by using Wilcoxon scores. Scores based on the Huber-function have also been suggested as a compromise between the Wilcoxon and the median test (Büning, 1997), but we have not found a noteworthy advantage over Wilcoxon scores here. Given the importance of the efficiency of the scale estimation we expect further improvements by incorporating information on previous scale estimates. Exponential smoothing is a natural candidate if the variability varies smoothly, while shift-preserving smoothers should be applied if the variability shows abrupt changes. Another possibility is to increase the left-hand window used for the scale estimation 
and the reference level, but then we need to rely on the level being approximately constant during longer time periods. Experiments not reported here show that a substantial increase in power is possible in this way with the ordering of the methods being essentially the same as reported above.

An issue not addressed in detail here is the action to be taken after a shift is detected. As opposed to the t-test and the ordinary rank tests the robustified rank tests indicate a level shift during several subsequent time points as long as the majority of the observations in one window is on a different level than the observations in the other window. As pointed out in Section 4, an estimate of the time point of the jump is needed if we want to use different level estimates before and after the shift. We might also want to reduce the window width close to the level shift for reducing the bias of the estimation there. This is especially important when using a longer left-hand window for comparison since we must only include observations coming from the same level in it.

Many more rules have been suggested for shift detection. Median comparisons also appear promising for locally constant, strongly contaminated time series. A closer investigation of such methods and a comparison to the robustified rank tests developed here is a task for further research. Robustified rank tests offer the advantage that they can easily be modified to detect abrupt shifts within monotonic trends. Replacing the median by Siegel's (1982) repeated median, we can fit a local linear trend to the data (Davies, Fried and Gather, 2004) and perform the tests on the residuals.

\section{Acknowledgements}

This work was prepared while the first author was working at the Department of Statistics of the University Carlos III of Madrid (Spain). The financial support of the Deutsche Forschungsgemeinschaft (SFB 475, "Reduction of complexity in multivariate data structures"), of the DAAD and the Minsterio de Educación ("Acciones integradas") is gratefully acknowledged. 


\section{References}

Bovik, A.C., Huang, T.S., Munson, D.C. Jr., 1986. Nonparametric tests for edge detection in noise. Pattern Recognition 19, 209-219.

Büning, H., 1997. Robust analysis of variance. J. Applied Statistics 24, 319-332.

Coakley, C.W., Hettmansperger, T.P., 1992. Breakdown bounds and expected resistance. J. Nonparametric Statistics 1, 267-276.

Croux, C., Rousseeuw, P.J., 1992. Time-efficient algorithms for two highly robust estimators of scale. In Dodge, Y., Whittaker, J. (eds.): Computational Statistics, Vol. 1, Heidelberg, Physica-Verlag, pp. 411-428.

Davies, P. L., Fried, R., Gather, U., 2004. Robust signal extraction for on-line monitoring data. J. Statistical Planning and Inference 122, 65-78.

Donoho, D.L., Johnstone, I.M., 1994. Ideal spatial adaptation by wavelet shrinkage. Biometrika 81, 425-455.

Fried, R., 2004. Robust filtering of time series with trends. J. Nonparametric Statistics 16, 313-328.

Gather, U., Fried, R., 2003. Robust estimation of scale for local linear temporal trends. Tatra Mountains Mathematical Publications 26, 87-101.

Grübel, R., 1988. The length of the shorth. Annals of Statistics 16, 619-628.

Hampel, F.R., 1974. The influence curve and its role in robust estimation. J. American Statistical Association 69, 383-393.

Lim, D.H., 2006. Robust edge detection in noisy images. Computational Statistics and Data Analysis 50, 803-812.

McKean, J.W., 2004. Robust analysis of linear models. Statistical Science 19, 562-570.

Nieminem, A., Neuvo, Y., Mitra, U., 1989. Algorithms for real-time trend detection. Signal Processing 18, 1-15.

Rousseuw, P.J., Croux, C., 1993. Alternatives to the median absolute deviation. J. American Statistical Association 88, 1273-1283. 
Rousseeuw, P.J., Leroy, A.M., 1988. A robust scale estimator based on the shortest half. Statistica Neerlandica 42, 103-116.

Siegel, A.F., 1982. Robust regression using repeated medians. Biometrika 69, 242244.

Staudte, R.G., Sheather, S.J., 1990. Robust Estimation and Testing. John Wiley \& Sons, New York.

Tukey, J. W., 1977. Exploratory Data Analysis. Addison-Wesley, Reading, Mass. (preliminary edition 1971).

Ylvisaker, D., 1977. Test resistance. J. American Statistical Association 72, 551556. 